\title{
TWO-SCALE CONVERGENCE IN THIN DOMAINS WITH LOCALLY PERIODIC RAPIDLY OSCILLATING BOUNDARY
}

\author{
IRINA PETTERSSON
}

Abstract. The aim of this paper is to adapt the notion of two-scale convergence in $L^{p}$ to the case of a measure converging to a singular one. We present a specific case when a thin cylinder with locally periodic rapidly oscillating boundary shrinks to a segment, and the corresponding measure charging the cylinder converges to a one-dimensional Lebegues measure of an interval. The method is then applied to the asymptotic analysis of linear elliptic operators with locally periodic coefficients and a p-Laplacian stated in thin cylinders with locally periodic rapidly varying thickness.

Mathematics subject classification (2010): Primary 35B27, secondary 74K10.

Keywords and phrases: Two-scale convergence, singular measure, homogenization, thin domain with varying thickness, oscillating boundary, dimension reduction, locally periodic operators, p-Laplacian.

\section{REFERENCES}

[1] G. Allaire, Homogenization and two-scale convergence, SIAM J. Math. Anal., 23, 6 (1992), 14821518.

[2] B. Amaziane, M. Goncharenko, and L. Pankratov, $\gamma d$-convergence for a class of quasilinear elliptic equations in thin structures, Mathematical methods in the applied sciences, 28, 15 (2005), $1847-1865$.

[3] N. Ansini AND ANdrea Braides, Homogenization of oscillating boundaries and applications to thin films, Journal d'Analyse Mathématique, 83, 1 (2001), 151-182.

[4] G. Aronsson, L. Evans, AND Y. Wu, Fast/slow diffusion and growing sandpiles, Journal of Differential Equations, 131, 2 (1996), 304-335.

[5] J. M. ARRIETA AND M. C. PEReIRA, Homogenization in a thin domain with an oscillatory boundary, J. Math. Pures Appl. (9), 96, 1 (2011), 29-57.

[6] J. M. ARrieta AND M. Villanueva-Pesqueira, Thin domains with non-smooth periodic oscillatory boundaries, J. Math. Anal. Appl., 446, 1 (2017), 130-164.

[7] D. Blanchard AND A. GaUdiello, Homogenization of highly oscillating boundaries and reduction of dimension for a monotone problem, ESAIM: Control, Optimisation and Calculus of Variations, 9 (2003), 449-460.

[8] D. BORISOV AND P. FREITAS, Asymptotics of dirichlet eigenvalues and eigenfunctions of the laplacian on thin domains in $\mathbf{R}^{d}$, Journal of Functional Analysis, 258, 3 (2010), 893-912.

[9] G. Bouchitté And I. Fragalà, Homogenization of thin structures by two-scale method with respect to measures, SIAM J. Math. Anal., 32, 6 (2001), 1198-1226 (electronic).

[10] G. Bouchitté, L. Mascarenhas, And L. Trabucho, Thin waveguides with Robin boundary conditions, J. Math. Phys., 53, 12 (2012), 123517, 24 p., 2012.

[11] G. Bouchitté, L. Mascarenhas, And L. Trabucho, On the curvature and torsion effects in one dimensional waveguides, ESAIM: Control, Optimisation and Calculus of Variations, 13, 4 (2007), 793-808.

[12] A. BRAIDES, I. FonseCA, AND G. FRANCFORT, 3D-2D asymptotic analysis for inhomogeneous thin films, Indiana Univ. Math. J., 49, 4 (2000), 1367-1404.

[13] Ph. Ciarlet And PH. Destuynder, A justification of a nonlinear model in plate theory, Computer methods in Applied Mechanics and engineering, 17 (1979), 227-258. 
[14] IoAnna-Andreea Ene And JeAnnine SAint-JeAn Paulin, Homogenization and two-scale convergence for a stokes or navier-stokes flow in an elastic thin porous medium, Mathematical Models and Methods in Applied Sciences, 6, 07 (1996), 941-955.

[15] L. Evans, Partial differential equations, Graduate Studies in Mathematics, 19, American Mathematical Society, Providence, RI (2010).

[16] L. Friedlander AND M. SOlOMyaK, On the spectrum of the Dirichlet Laplacian in a narrow strip, Israel J. Math., 170 (2009), 337-354.

[17] A. Piatnits Ki G. Chechin and A. Shamaev, Homogenization methods and applications, 234, American Mathematical Society, Providence, RI (2007).

[18] A. Gaudiello, B. Gustafsson, C. Lefter, J. Mossino, ET Al., Asymptotic analysis for monotone quasilinear problems in thin multidomains, Differential and Integral Equations, 15, 5 (2002), 623-640.

[19] B. GustafsSon AND J. Mossino, Non-periodic explicit homogenization and reduction of dimension: the linear case, IMA Journal of Applied Mathematics, 68, 3 (2003), 269-298.

[20] S. MARUŠIĆ AND E. MARUŠIĆ-PALOKA, Two-scale convergence for thin domains and its applications to some lower-dimensional models in fluid mechanics, Asymptotic Analysis, 23, 1 (2000), 23-57.

[21] T. A. MeŁniK And A. V. Popov, Asymptotic analysis of boundary value problems in thin perforated domains with rapidly changing thickness, Nelını̄nı Koliv., 13, 1 (2010), 50-74.

[22] F. Murat And A. Sili, Problemes monotones dans des cylindres de faible diametre formés de matériaux hétérogenes, Comptes Rendus de l'Academie des Sciences-Serie I-Mathematique, 320, 10 (1995), 1199-1204.

[23] S. A. Nazarov, E. PÉrez, And J. TASKinen, Localization effect for Dirichlet eigenfunctions in thin non-smooth domains, Trans. Amer. Math. Soc., 368, 7 (2016), 4787-4829.

[24] S. A. NAZArov, Asymptotic Theory of Thin Plates and Rods, vol. 1 (in Russian), Novosibirsk: Nauchnaya Kniga (2001).

[25] G. NGUETSENG, A general convergence result for a functional related to the theory of homogenization, SIAM J. Math. Anal., 20 (3): 608-623, 1989.

[26] G. PANASEnKo, Multi-scale modelling for structures and composites, Springer, Dordrecht (2005).

[27] I. PANKRATOVA AND A. PiATNITS KI, Homogenization of spectral problem for locally periodic elliptic operators with sign-changing density function, J. Differential Equations, 250, 7 (2011), 3088-3134.

[28] I. PANKRATOVA AND K. PetTERSSON, Spectral asymptotics for an elliptic operator in a locally periodic perforated domain, Appl. Anal., 94, 6 (2015), 1207-1234.

[29] M. C. PEREIRA AND R. P. SiLva, Error estimates for a Neumann problem in highly oscillating thin domains, Discrete Contin. Dyn. Syst., 33, 2 (2013), 803-817.

[30] M. C. Pereira AND R. P. Silva, Remarks on the p-laplacian on thin domains, Contributions to nonlinear elliptic equations and systems, Progr. Nonlinear Differential Equations Appl., 86 (2015), 389-403.

[31] L. Rudin, S. Osher, AND E. FATEMI, Nonlinear total variation based noise removal algorithms, Physica D: Nonlinear Phenomena, 60, 1-4 (1992), 259-268.

[32] R. P. SILVA, Behavior of the p-laplacian on thin domains, Int. J. Differ. Equ., Article ID 210270:2013 (2013), 4 pp.

[33] V. ZHIKOV, On an extension and an application of the two-scale convergence method, Mat. Sb., 191, 7 (2000), 31-72.

[34] V. V. Zhikov, On two-scale convergence, Tr. Semin. im. I. G. Petrovskogo, 23, 410 (2003),149-187. 